\title{
Unrestrained Multiple-Sensor System for Bed-Leaving Detection and Prediction
}

\author{
Hirokazu Madokoro*, Nobuhiro Shimoi, Kazuhito Sato \\ Faculty of Systems Science and Technology, Akita Prefectural University \\ 84-4, Tsuchiya Aza Ebinokuchi, Yurihonjo City, Akita, 015-0055, Japan
}

\begin{abstract}
Copyright (C) 2015 by authors, all rights reserved. Authors agree that this article remains permanently open access under the terms of the Creative Commons Attribution License 4.0 International License
\end{abstract}

\begin{abstract}
This paper presents an unrestrained sensor system that analyzes predictive behavior patterns that occur when a patient leaves a bed. Our system comprises three sensors: pad sensors installed under a bed mat, a pillow sensor to detect head movements, and a bolt sensor mounted to a bed handrail. We used a triaxial accelerometer for a pillow sensor and piezoelectric elements for the pad sensors and bolt sensor. The salient features of these sensors are their easy installation, low cost, high reliability, and toughness. Moreover, we developed a method of recognizing bed-leaving behavior patterns using machine-learning algorithms from signals obtained using the sensors. We evaluated our system by examining ten subjects in an environment representing a clinical site. The mean recognition accuracy for seven behavior patterns was $75.5 \%$. Furthermore, the recognition accuracies for longitudinal sitting, terminal sitting, and left the bed were $83.3 \%, 98.3 \%$, and $95.0 \%$, respectively. In contrast, falsely recognized patterns remained inside of respective categories of sleeping and sitting. We believe that our system is applicable to an actual environment as a novel sensor system with no restraint of patients.
\end{abstract}

Keywords Bed-Leaving, Machine Leaning, Piezoelectric elements, Accelerometer, and Quality of Life

\section{Introduction}

Along with the longevity of the society, labor shortages will become severe, especially at nursing-care facilities [1]. Few caretakers must care for numerous aged patients. For this situation, caretakers monitor them inadequately, especially during sleep at night [2]. Mitadera et al. reported that approximately half of accidents are falling or tumbling among aged patients [3]. Numerous accidents occurred when they leave their bed. Moreover, these accidents occurred in a place where caretakers are hard to keep their eye on. The accident rates of tumbling and falling were $83.3 \%$ and $85.4 \%$, respec- tively, when they were not supported at the moment of accidents. For protecting these accidents, it requires suitable provision after conducting assessments for respective patients. One approach to this problem is to use bed-leaving sensors that signal when patients leave from their beds. They can be used to prevent falling from their beds. The number of hospitals and nursingcare facilities using these sensors has increased $[4,5]$.

Actually, clip sensors are the lowest-cost sensors that can be introduced easily. This is a simple sensor attached to a patient's clothing [6]. According to protection of human rights, the usage of clip sensors has been prevented recently because it requires constraint of the wearer. For the performance of clip sensors, malfunctions and anomaly detections occur frequently because of the binary response used to detect bed-leaving behaviors. Regarding the reliability and perspective of management, clip sensors are insufficient to prevent falling from a bed completely. Moreover, accidents caused by binding of the neck in a cable have been reported [6]. We regard clip sensors as inadequate for use at clinical sites, although it is easy to introduce them at low cost.

Recently, mat sensors are widely used as a low-cost and convenient sensors that can be installed easily [7]. Haruyama et al. developed an alarm system to detect patients leaving from their beds using mat sensors [8]. Medical and welfare suppliers released various mat sensors installed on a floor, a bed, or on rolling handrails. Mat sensors used on a floor are unnecessary for authentication for medical devices under the pharmaceutical law. Regarding the performance of detection, a problem of a delay remains because of the response after sitting at the end of the bed, although such sensors are easy to produce and to sell. Moreover, sensor responses are apparent when medical staff members such as a nurse or a medical doctor walk on the mat. To distinguish the responses of patients and medical personnel is a challenging problem for signal pattern recognition. Sensors rolled over handrails not only obstruct a view of a bed, but also present a risk of removal of a sensor when a patient finds it and feels negatively about being restrained. Furthermore, false detection occurs when patients leave their bed without gripping a handrail. Mat sensors in- 
stalled on a bed can detect bed-leaving with higher reliability than other mat sensors. However, existing mat sensors are actuated by a binary response similar to that for clip sensors. Early detection is not realized, especially in the initial stage of bed-leaving behavior.

Using a camera as a bed monitoring sensor can provide a low-cost system. Moreover, it can obtain much information for a subject. However, it is a challenging task to predict behavior patterns obtained from images, even when state-of-the-art computer vision technologies are used. For this method, medical staff members must observe images directly. It is impossible to monitor numerous subjects simultaneously with a few operators. Moreover, we must consider aspects of human rights and quality of life (QOL). Especially, it is impossible to recognize behavior patterns related to bed-leaving using only sensor responses, even when detailed analyses are conducted, because behavior patterns differ among people [9]. Moreover, monitoring using a camera imposes a mental load on patients because they feel as though they are under surveillance all day and all night.

For solving these problems, this paper presents an unrestrained sensor system that analyzes predictive behavior patterns that occur when a patient leaves a bed. We developed a machine-learning-based method that obviates the setting of thresholds in advance. Moreover, we developed an integrated system that can send data obtained from sensors to a monitoring computer using a close-range wireless module. Our former approach remained a problem for reliable detection and prediction because we merely used only pad sensors [10]. For this paper, we propose a novel multiple-sensor system to improve accuracy according to a usage of a target. We intend to reduce the amount of data used for predicting and minimizing incorrect recognition given a minimum number of sensors. We evaluated our sensor system in an environment that represents a clinical site. Results show that our method predicted seven behavior patterns related to bed-leaving, especially for longitudinal sitting and terminal sitting.

\section{Related Work}

Large-scale systems using numerous sensors of various types have been proposed for prediction at the initial stage to measure behavior patterns in detail. Shimizu et al. proposed a bed-leaving detection system using ultrasonic array sensors [11]. They evaluated their system at a hospital as a demonstration experiment. Hirasawa et al. proposed a method to expose infrared rays to the upper part of the bed as a system to prevent falling accidents [12]. Uezono et al. proposed a large-scale monitoring system for detecting bed-leaving behavior patterns using 96 strain gauges assigned for a reticular pattern [13]. These large-scale sensor systems can realize higher accuracy and more stable detection than low-cost sensors, such as clip sensors or mat sensors, can. However, these sensors are not put into practical use because of their cost. Moreover, high expenditures are necessary to replace a bed or for construction for installation whenever these systems are improved for practical use in a market.
Hatsukari et al. developed a bed-leaving detection system using strain gauges installed inside of actuators to obtain weight changes of a person on a bed [17]. They embedded sensors and a controller to Paramount beds as a new product of their company Paramount Bed Co. Ltd. The detection accuracies for longitudinal sitting and terminal sitting were, respectively, $87.0 \%$ and $98.1 \%$ [17]. For their system, three actuators are used in a bed. Each actuator has four biaxial strain gauges installed on diagonal lines. We regard this as the most popular bedleaving sensor system in practical use currently. It has both high performance and reliability. However, an important limitation of this system is that it is used only for the lifting beds of that company's products with installed actuators. Moreover, it is necessary to install sensors into actuators at the manufacturing phase of beds. Therefore, users must buy it if they need to use this function of bed-leaving detection. These are not casually used sensors that can be installed later. In addition, strain gauges used for this system have uncertain response to thermal changes. For characteristic variation caused by thermal changes, they described that they accommodated it with software. Moreover, this system requires that the weight setting of a person to be selected from three divisions in advance.

Kishimoto et al. proposed a method to predict poses using a capacitance triaxial acceleration sensor to detect transition points in sleeping automatically [18]. For automatic detection of pose transition points, they used threshold-based processing to apply first derivation to sensor outputs. Using $k$-means as a non-hierarchical clustering method for pose prediction, their method detected five behavior patterns: face-up sleeping, right sleeping, left sleeping, face-down sleeping, and sitting. For their evaluation experiment using datasets obtained from 13 subjects, the mean recognition accuracy was $90.96 \%$. However, it is necessary for restriction because this sensor is installed near the epigastric fossa. Regarding clinical applications as the conditions of actual use, we consider that it is difficult to assent to wearing the sensor. Moreover, they set thresholds empirically for detecting behavior transition points. We consider that maintaining accuracy while absorbing individual differences is a challenging task.

Regarding quality of sleep (QOS), Imoto et al. measured body motion during sleeping using a watch-shaped triaxial acceleration sensor. Their method detected body motion at one minute intervals in bandpass signals of $2-3 \mathrm{~Hz}$ acceleration changes using the threshold of $\pm 0.01 \mathrm{G}$. They evaluated the method for one week using datasets obtained from six subjects in their 50 s who were diagnosed as needing daily life guidance. Their method recognized sleeping or arousal for timeseries active mass datasets. They used experimentally obtained results for each subject to assess sleeping parameters quantitatively. However, the results failed to demonstrate a global tendency in light of relations to the number of temporary arousals during sleep. Moreover, we regard that this watch-shaped sensor decreases QOS when worn on an arm during sleeping because its use requires that a subject be restrained.

As a monitoring system with unrestrained sensors, Okada et al. proposed a method to install an accel- 


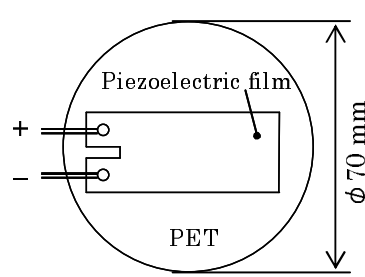

(a) Upper view

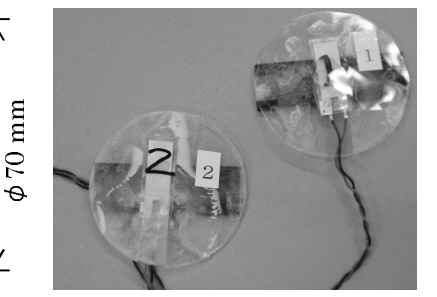

(b) developed sensors
Figure 1. Design and prototype of pad sensors.

eration sensor in a comforter cover [20]. This system was constructed to measure the heart rate of a subject during sleeping. Near the position of the heart in a subject, this sensor was stitched to the surface of a cover on the upper-left chest region. The acceleration sensors using piezoelectric ceramics have advanced sensitivity. They obtained measurement results resembling the waves of an electrocardiogram (ECG) during faceup sleeping under resting status. The signal peak of acceleration changes was obtained in the corresponding position of the $\mathrm{R}$ wave on the ECG. Moreover, the signal peaks, which were similar to peaks of the $\mathrm{R}$ wave, were counted to assess the side sleeping behavior. The compact sensor weight was only $4 \mathrm{~g}$. We regard this system as also requiring amplifier circuits and A/D convertors to capture signals for processing in a computer, but their paper veiled many details of the system. We regard wiring of the sensor to a comforter cover as a remaining obstacle to practical use of this device.

\section{Multiple sensor system}

High-performance and functional sensors of various types were used for existing bedside monitoring systems for targeting expensive care or medical treatments $[11,12,13]$. Alternatively, it is necessary to attach them to the body to realize steady and long-term monitoring $[19,20]$. For this study, we designed a sensor system that is practical, convenient, inexpensive, and simple. We specifically examine the following features: 1) a bed mat to distribute the body weight of a subject directly, 2) a pillow to distribute the weight of the head, and 3) a handrail that a subject can grip while standing immediately before leaving. For multiple sensing, our system comprises following three sensors: 1) a pad sensor under the bed mat, 2) a pillow sensor to detect acceleration according to head movements, and 3) a bolt sensor used for a connecting a point of a handrail. We explain features in each sensor and the overall system architecture as the following.

\subsection{Pad sensor}

Our originally developed plate-shaped sensors can be installed easily under a bedsheet. Fig. 1(a) depicts the design structure and an overview photo of our prototype sensors [14]. The shape of pad sensors is roundness because it can detect wight changes from all directions. As a prototype, we used piezoelectric piezoelectric films by Measurement Specialties Inc [21]. We fixed a piezoelec-

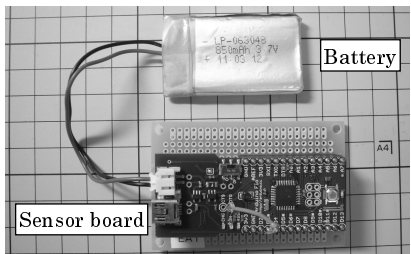

(a) Sensor board and battery

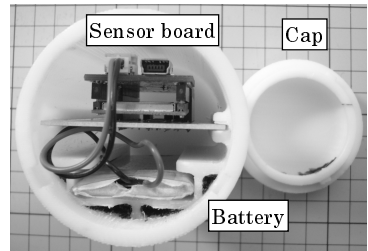

(b) Installed in the case
Figure 2. Sensor board, Lithium-ion polymer battery, and their storage capsule. The sensor board and capsule are originally developed for this studv
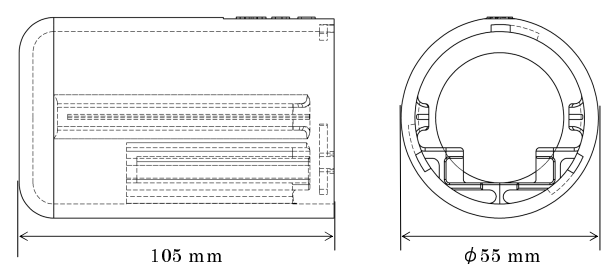

Figure 3. Design of the capsule stored for a microprocessor board with an accelerometer and a battery.

tric film between two polyethylene terephthalate (PET) plates of laminated polyester. The polyester and PET plate sizes were, respectively, $125 \mu \mathrm{m}$ and $\phi 70 \times 0.5 \mathrm{~mm}$. Figs. 1(b) depicts the design structure and an overview photo of our prototype sensors.

Output voltage is generated from the bent piezoelectric films when a subject transfers body weight on the bed.This sensor can measure recursively because the reference potential is offset when the bending stops. Moreover, the strength of weight according to changes of the body is obtainable linearly because the bend of the piezoelectric film and output voltage has a relation of proportionality. Furthermore, piezoelectric films are less troublesome and provide no false operations because they have simple wiring without electric power supply for measurements. Additionally, we can provide a lowcost system requiring no maintenance related to replacement of a battery.

\subsection{Pillow sensor}

For our pillow sensor, we developed a triaxial acceleration sensor module MMA7361LC produced by Freescale Semiconductor Inc. Fig. 2(a) depicts the exterior of our original board. Herein, we evaluated basic characteristics of this sensor module though our former study of attitude control for a flying robot [15].

As the power source, we used a lithium-ion polymer battery with 0.85 Ah capacity. Additionally, we designed a case used for storing the board and the battery to a pillow. Fig. 3 depicts design parameters of the case. This is a 55 -mm-diameter, 105 -mm-long cylindrical case. We made it from acrylonitrile butadiene styrene (ABS) using a three-dimensional printer. The board and battery are stored to the upper and lower part in the case using sliding rails. We set no margin for the slides because the sensor boards were fixed inside of the case. Fig. 2(b) presents a photograph of the device installed in the case. As shown on the left side of the photograph, after storing the device, we put on a cap for sealing. 


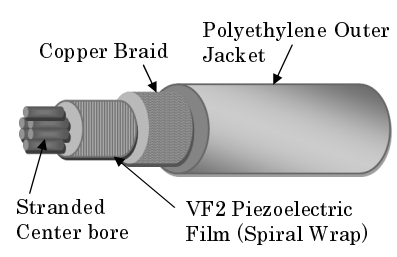

(a) Inside structure

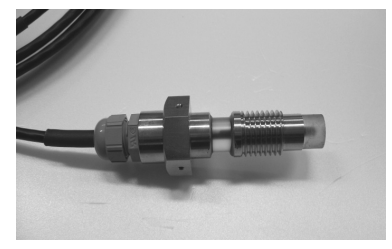

(b) Developed sensor
Figure 4. Design and prototype of bolt sensors.

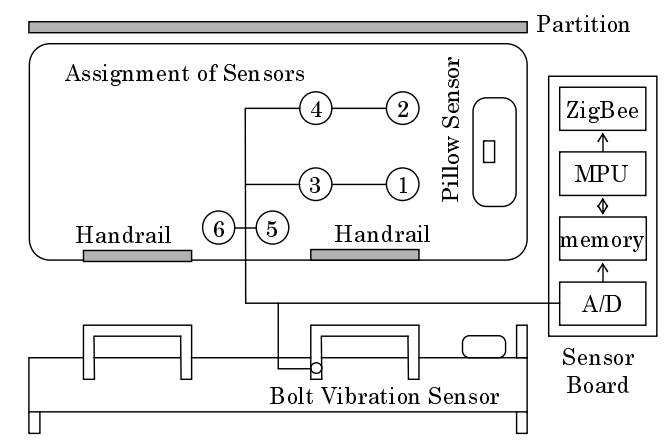

Figure 5. Block diagram of our proposed system and assignment of our developed sensors on the bed. The symbols of $1-6$ correspond to the sensor assignment of S1-S6.

\subsection{Bolt sensor}

We use a bolt sensor for the joint between a bed and a handrail. We assume that this sensor conducts overall sensing of a bed, whereas pad sensors are used for partial sensing of a subject. Moreover, this sensor detects weight changes that occur when a subject tries to stand up and grip a handrail. Fig. 4(b) portrays a bolt sensor that we developed as a prototype. This sensor was developed originally by Shimoi et al. [16] as a simple sensor to measure bridge strain. This sensor has been commercially available for some time. For this study, we originally developed a sensor with $\phi=10 \mathrm{~mm}$ to fit the size of the bolt to the joint of the bed used for our evaluation experiment.

Fig. 4(a) depicts the sensor structure. We inserted a piezoelectric cable into hollow polyurethane resin. Subsequently, we attached a bolt-shaped adapter as a cover for the exterior. Using this adapter, the bolt sensor can be connected to a nut. We extracted wires to obtain sensor signals, although no electric power source is necessary for piezoelectric cables. The datasheet shows that piezoelectric cables generate voltage linearly according to bending. Moreover, we confirmed this feature through our preliminary experiment and earlier experiments reported by Shimoi et al. [16].

\section{4 system structure}

Figure 5 portrays the entire structure of our sensor system that we originally developed for this study. Our system comprises six pad sensors installed over the bed, one bolt sensor mounted on the joint part between a handrail and a frame of the bed, and one pillow sensor: an acceleration sensor installed inside of a pillow.

The assignment of six pad sensors is S1 and S2 for the shoulder part, S3 and S4 for hip part, and S5 and $\mathrm{S} 6$ for the terminal part. We assigned these six sensors

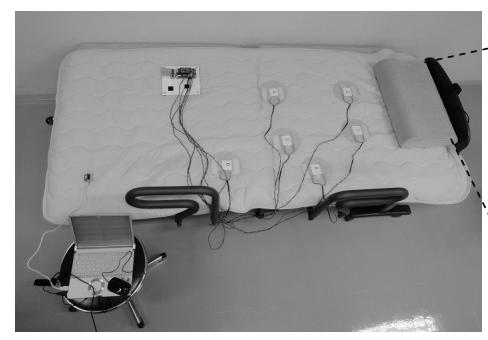

(a) Whole system

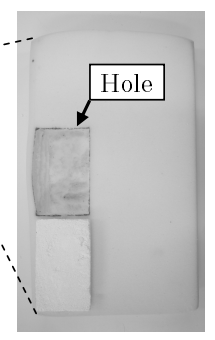

(b) Pillow
Figure 6. Photographs of the bed and pillow used for this study.

referring to the literature related to the development of a monitoring system for users of welfare care beds [4, 5]. We mounted a bolt sensor to the joint of a handrail on one side of the bed. The size of the bed used for this experiment is $210 \mathrm{~cm}$ long, $100 \mathrm{~cm}$ width, and 70 $\mathrm{cm}$ height. This bed has an actuator for reclining the back pannel. For this experiment, we did not use this function.

We used a commercially available pillow for embedding the sensor. This low-resilience urethane pillow purchased from Nitori Co. Ltd. was $50 \mathrm{~cm}$ wide, $31 \mathrm{~cm}$ deep, with $9 \mathrm{~cm}$ height. We cut out the back side of the pillow shown in Fig. 5(b) to produce a void space to insert the sensor case. We fixed the sensor case with three points using a hook and loop fastener to prevent rolling inside the pillow. One advantage of our sensor system is its easy installation as an aftermarket add-on part, although it is necessary to open a pillow and partially empty it during repairs. Moreover, the sensor system requires no calibration. Regarding the place to store the sensor, we were concerned that it would feel uncomfortable or decrease the feature of cushioning when putting the head to the pillow. Subjects participating in the experiment reported no negative impression from using this pillow.

The primary feature of our system is that it realizes monitoring using unrestrained sensors. We consider QOL for a patient to live life normally. Our system requires no supervision using infrared cameras or constraining sensors such as clip sensors. Moreover, we can create a low-cost system using piezoelectric films as sensors that can function with little trouble or missed operations, and with remarkable characteristics for pressure resistance. This board obtains output voltage from each sensor for wireless communication. We developed this board using Open Source Hardware ArduinoFIO. With consideration of power consumption, we used shortrange wireless communication standard ZigBee communicated with a monitoring terminal computer. Measured signals are displayed to a monitoring computer in real time. We embedded our method based of machine learning to this software for bed-leaving prediction.

\section{Prediction Method}

\subsection{Target behavior patterns}

The target behavior patterns for bed-leaving prediction comprise three groups: sleeping, sitting, and leaving. For this study, we attempt to classify detailed be- 


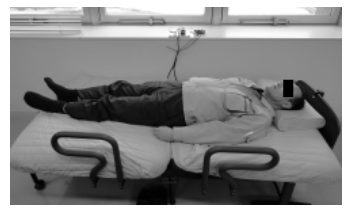

(1) Face-up sleeping (FU)

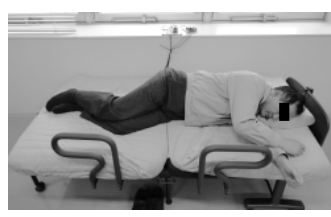

(3) Left sleeping (LE)

(5) Lateral sitting (LA)

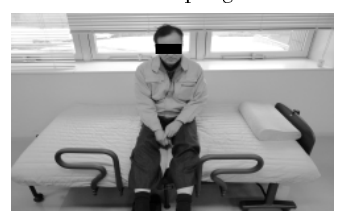

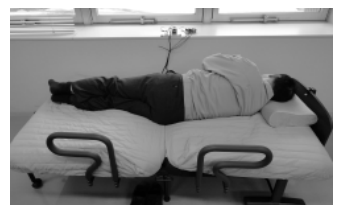

(2) Right sleeping (RI)

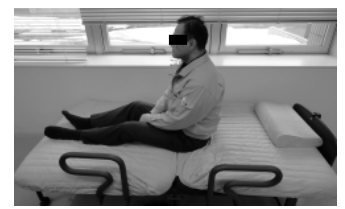

(4) Longitudinal sitting (LO)

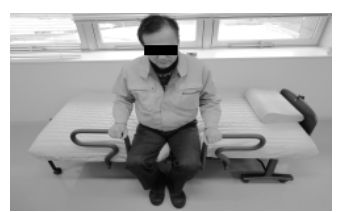

(6) Terminal sitting (TE)

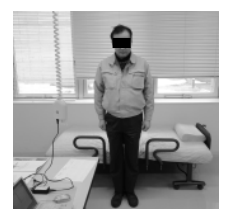

(7) Left the bed (LB)

Figure 7. Photographs of target patterns in each body position on the bed.

havior patterns from the responses of six sensors. The sleeping comprises three patterns: face-up sleeping, left sleeping, and right sleeping. The sitting comprises three patterns: longitudinal sitting, lateral sitting, and terminal sitting [24]. The total prediction target is to produce seven patterns including leaving. Fig. 7 depicts photographs in each pose for the target behavior patterns. The following are features and estimated sensor responses in each pattern.

1. Face-up sleeping (FU): a subject is sleeping on the bed normally to the upper side of the body.

2. Right sleeping (RI): a subject is rolling over on the bed to the right side.

3. Left sleeping (LE): a subject is rolling over on the bed to the left side. These three patterns occur as sleeping behaviors. The following are the target patterns of bed-leaving prediction.

4. Longitudinal sitting ( $\mathbf{L O}$ ): a subject is sitting longitudinally on the bed after rising.

5. Lateral sitting (LA): a subject is sitting laterally on the bed after turning the body from longitudinal sitting.

6. Terminal sitting (TE): a subject is sitting in the terminal position on the bed trying to leave the bed. Rapid and correct detection are necessary because of the terminal situation for leaving the bed.

7. Left the bed (LB): a subject is left the bed. Herein, sensor responses disappear in the status of

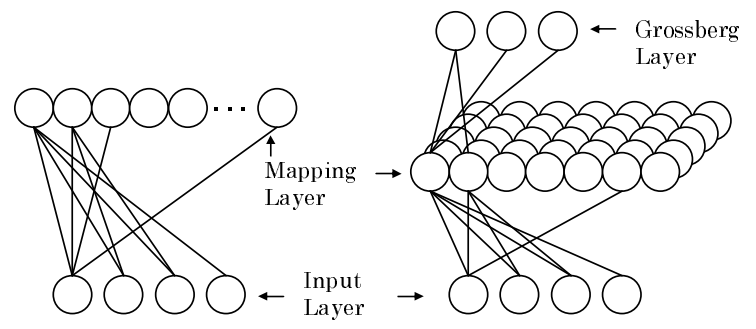

(a) Self-Organizing Maps

(b) Counter Propagation Networks

Figure 8. Network architecture of SOMs and CPNs.

losing consciousness or a life crisis such as cardiopulmonary arrest. For such circumstances, monitoring devices such as electrocardiographs are used. Therefore, such circumstances are beyond our prediction targets.

Longitudinal sitting is just a behavior pattern by which a subject sits up. In numerous cases, subjects will return to sleeping. In lateral sitting, a subject will move to leave from their bed because they move to turn the body to the terminal. Therefore, our system must determine lateral sitting immediately to predict a subject leaving the bed. Moreover, rapid and correct detection is necessary because of the terminal situation for leaving the bed if a subject moves to longitudinal sitting. We consider that our system can protect patients from injury or accidents caused by falling from the bed because it can detect such phenomena before a subject leaves completely.

\subsection{Preprocessing}

Actually, original signals include much more noise, which decreases the recognition accuracy and increases calculation costs. As a method to remove noise signals, filters set by thresholds have been used widely for preprocessing. However, calibration or resetting is necessary according to a target because thresholds are set subjectively or empirically in most cases. Moreover, it is a difficult task to absorb characteristic variation of sensors used only for fixed thresholds. For targeting humans as a measurement objective, individual variations have strong effects.

To set a body weight of a subject in advance is commonly used as a solution against this problem [17]. The load changes are strongly related to body weight. We consider that it is insufficient for calibration used only for body weight because behavior patterns vary among sexual and age differences. In contrast, we are aiming at developing an approach for bed-leaving prediction without setting thresholds in advance. For this study, we use machine-learning algorithms dealing with individual differences among subjects. Nakamura et al. described the effectiveness for adaptation of individual differences using machine-learning methods [23]. Similarly, our method is necessary without setting thresholds through learning patterns in each subject from original signals obtained using sensors.

Various methods have been proposed for machinelearning algorithms. For this study, we use SelfOrganizing Maps (SOMs) [25], which learn similarities 
of input data through the process of learning. Based on the concept of neighborhood and competitive learning, SOMs create clusters using self-mapping characteristics of unsupervised learning. Using the advanced calculation performance of computers, SOMs have facilitated various applications such as remote sensing, facial image processing, and character recognition. For actual applications, SOMs are validated as effective and superior performance through various existing studies [26].

Actually, $k$-means [27] is widely used for unsupervised-learning based clustering. Vesanto et al. validated that SOMs have superior performance to that of $k$-means through their experiments [28]. Moreover, Terashima et al. demonstrated that false recognition accuracy of SOMs decreased to the minimum compared with $k$-means used for clustering. Therefore, we used SOMs because of these benefits.

Fig. 8(a) depicts a network architecture of SOMs. The network comprises two layers: an input layer and a mapping layer. Units on the input layer are assigned as the number of features of input data. We set two units because we used a triaxial acceleration sensor for this system. The mapping layer consists of units in a lowdimensional space. In our method, we assigned mapping units for one dimension because we used it for vector quantization on clustering. Learning is conducted to burst a unit on the mapping layer when a set of input signals is given.

The learning algorithm of SOMs is the following. $w_{i, p}(t)$ are weights from an input layer unit $i(i=1 \ldots, I)$ to a mapping layer unit $p(p=1 \ldots, P)$ at time $t$. These weights are initialized randomly before learning. The Euclidean distance $d_{p}$ between $x_{i}(t)$ and $w_{i, p}(t)$ is calculated as

$$
d_{p}=\sqrt{\sum_{i=1}^{I}\left(x_{i}(t)-w_{i, p}(t)\right)^{2}} .
$$

The unit for which $d_{p}$ is the smallest is sought as the winner unit $c$.

$$
c=\operatorname{argmin}\left(d_{p}\right) .
$$

The neighborhood region $N_{p}(t)$ around $c$ is defined as

$$
N_{p}(t)=S\left(1-\frac{t}{O}\right) .
$$

Therein, $S(0<S \leq P)$ is the initial size of $N_{p}(t) ; O$ is the maximum iteration for training. Subsequently, $w_{i, p}(t)$ of $N_{p}(t)$ are updated to close input feature patterns.

$$
w_{i, p}(t+1)=w_{i, p}(t)+\alpha(t)\left(x_{i}(t)-w_{i, p}(t)\right)
$$

Therein, $\alpha(t)$ is a learning coefficient that has decreasing value with the progress of learning as

$$
\alpha(t)=\alpha(0)\left(1-\frac{t}{O}\right)
$$

where $\alpha(0)(0<\alpha(0) \leq 1.0)$ is the initial value. In the initial stage, the learning speed is fast when the rate is high. In the final stage, the learning converges while the rate decreases.
During the learning phase, the process above is repeated until $O$ to pick up from training datasets for random sampling. After learning, training datasets are presented again to the network for searching burst units. These units interpret labels against training datasets and share the same labels as clusters. Herein, for our sensor system using piezoelectric elements, output voltages near the offset are given when no load is changed. The range around the voltages is mapped to numerous signals. We can estimate the appearance of a unit that corresponds to the maximum number of signals on the category map. Therefore, we can select invalid signals that are necessary for recognition to remove invalid signals corresponding to the unit.

\subsection{Recognition method}

After removing noise from original signals, we recognize behavior patterns using supervised learningbased methods. The purposes of supervised learning and unsupervised learning are to acquire information representation[31] and to create mapping relations[30]. Popularly used methods for supervised learning are Support Vector Machines (SVMs), which provide advanced performance with mapping to a high dimensional space using a kernel function, or Boosting, which is a method combining numerous weak learning machines. For this study, we use Counter Propagation Networks (CPNs) [32], a supervised learning algorithm to be expanded based on SOMs of unsupervised learning.

We use CPNs, not SVMs or Boosting, because of the following two features. 1) The learning algorithm is easy to implement for inserting a Grossberg layer to SOMs that we used for preprocessing. 2) Relations among signals are visualized though the process of creation of mapping structures. We describe formulas of the learning algorithms on CPNs in different parts of SOMs. Visualization of relations among signals is actualized on a category map [26]. We particularly present visualization of results obtained using figures at the evaluation experiment.

The CPNs proposed by Nilsen [32] contain the network structure to append a Grossberg layer, which is given teaching signals, as the third layer. Fig. 8(b) depicts the network architecture of CPNs. The input and mapping layers of CPNs are similar to SOMs. The Grossberg layer is assigned to the counter position of the input layer. For this study, we selected the twodimensional mapping layer for visualization of similarity among features of input data.

The CPN learning algorithm is the following. The algorithm from presenting input data through updating weights after searching the winner unit $c$ consists of the similar procedure of SOMs. However, weights and neighborhood regions are changed respectively to $w_{i, p, q}(t)(p=1 \ldots, P, q=1 \ldots, Q)$ and $N_{p, q}(t)$ because of the use of a two-dimensional mapping layer. The $v_{j, p, q}(t)$ are weights from a Grossberg layer unit $j(j=1 \ldots, J)$ to a mapping layer unit $(p, q)(p=1 \ldots, P ; q=1 \ldots, Q)$ at time $t$. $v_{i, p, q}(t)$ and its neighborhood units inside $N_{p, q}(t)$ are updated based on the following Grossberg learning algorithm.

$$
v_{j, p, q}(t+1)=v_{j, p, q}(t)+\beta(t)\left(T_{j}(t)-v_{j, p, q} j(t)\right),
$$


where $T_{j}$ represents teaching signals. Similarly to $\alpha(t)$, $\beta(t)$ is a learning coefficient that decreases its value with the progress of learning as

$$
\beta(t)=\beta(0)\left(1-\frac{t}{O}\right),
$$

where $\beta(0)(0<\beta(0) \leq 1.0)$ is the initial value. Finally, as the maximum value of $v_{j, p, q}(t)$ for the Grossberg layer unit $j$, label $L_{j}(t)$ is searched for the following.

$$
L_{j}(t)=\underset{1 \leq j \leq J}{\operatorname{argmax}}\left(v_{j, p, q}(t)\right) .
$$

After labeling all units, a category map is created as a learning result. Subsequently, datasets for testing are given to the network. A mapping layer unit is bursted as the minimum of the Euclidean distance in the formula (1). Results of CPNs are presented for corresponding labels.

\section{$5 \quad$ Experimental results}

\subsection{Experimental setup}

We set up an experimental environment similar to a clinical site for evaluation of our developed sensor system. We used a bed depicted in Fig. 7 with a subject. For this study, the number of subjects is ten persons: Subjects A-J. As attribute information, the body weights of the subjects are from $50 \mathrm{~kg}$ though 80 $\mathrm{kg}$. They repeated the behavior sequences of seven patterns for five times. Therefore, we obtained 35 pattern datasets in each subject. Each behavior is switched in $20 \mathrm{~s}$ intervals. We set the sampling rate for capturing signals to $50 \mathrm{~Hz}$. Herein, the number of subjects of our former study was only three persons [14].

Table 1 portrays setting parameters used in SOMs and CPNs. We determined these values based on our former study [33] and the literature by Hosokawa et al. [34]. As an evaluation method, we use leave-one-out Cross Validation (CV) [35]. For this experiment, four datasets and the remaining one dataset were used, respectively, for training and testing. Therefore, we evaluated our method for five combinational patterns in each subject.

\subsection{Measured signals}

Fig. 9 depicts output values of time-series signals obtained from nine sensors. The vertical and horizontal axes respectively depict the output voltage and transition time. The dashed lines depict boundaries of each behavior pattern. Output signal pattens differ in each subject.

For pad sensors, the range of output voltage on S1 though $\mathrm{S} 4$ was expanded, especially in the status of faceup, right, and left sleeping. Salient output voltages were given from S5 and S6, although they include some noise. The output voltage on S3 becomes high for longitudinal sitting. We consider that the body weight is concentrated to the hip area according to the rising behavior. For terminal sitting, the output voltage was given from S5 and S6. No output voltage was given from S1, S2, and S3. Moreover, S4, which is located near S5, gave

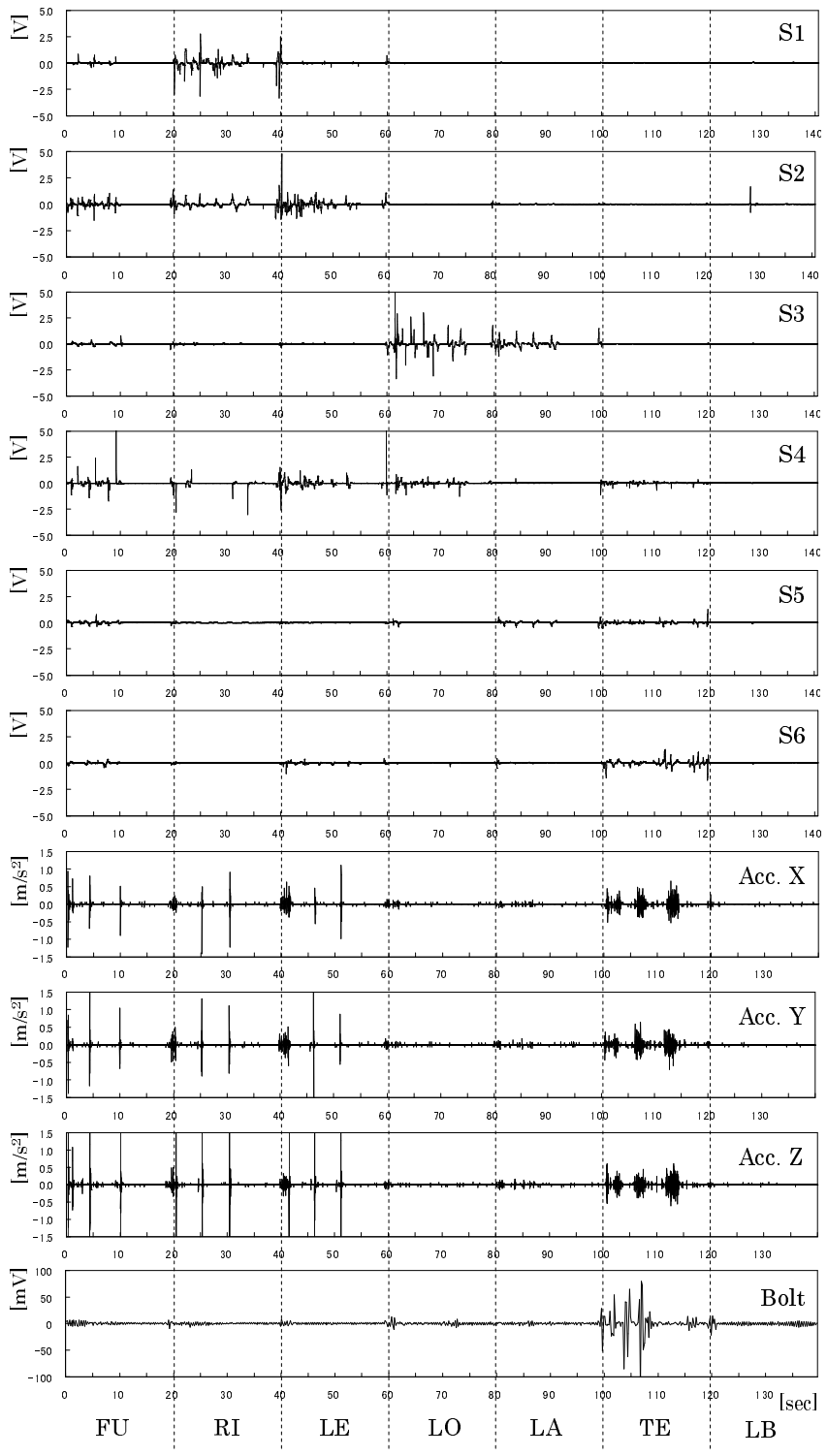

Figure 9. Time-series changes of sensor output in each behavior pattern (Subject A, first trial).

output voltage slightly. After leaving the bed, no output voltage was given from any sensor.

For the pillow sensor, the $\mathrm{Z}$ axis output of the upper and lower directions is greater than that of either the $\mathrm{X}$ or $\mathrm{Y}$ axis. Apart from the pillow, the output became small during longitudinal sitting after raising of the body of the subject. This tendency is similar to that of lateral sitting. For terminal sitting, the sensor output became wide because the subject tried to stand up at the terminal of the bed. We consider that this widening of output results from the behavior by which the subject tried to grip the safety handrail. Moreover, we consider that the weight shift at the bed terminal produces severe shaking all over the bed compared with that occurring around the center.

The signal outputs of the bolt sensor is presented only the terminal sitting. The bolt sensor captures slight strain. The width of output signals is $\pm 0.1 \mathrm{~V}$. The signal-noise rate is high compared with other two sensors.

This is the dataset of Subject A at the first trial. Output patterns of signals and their intensity differ in each 
Table 1. Setting values of parameters on SOM and CPN for this experiment.

\begin{tabular}{c|c|c|c|c|c|c|c|c}
\hline Method & $\mathrm{I}$ & $\mathrm{J}$ & $\mathrm{P}$ & $\mathrm{Q}$ & $\mathrm{S}$ & $\alpha(0)$ & $\beta(0)$ & $\mathrm{O}[\mathrm{epoch}]$ \\
\hline SOM & 10 & - & 10 & - & 16 & 0.5 & - & $1,000,000$ \\
\hline CPN & 10 & 7 & 20 & 20 & 16 & 0.5 & 0.8 & 100,000 \\
\hline
\end{tabular}

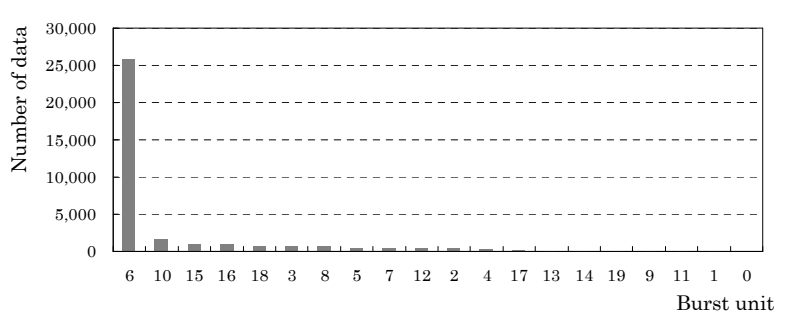

Figure 10. Histogram of classfication results obtained using SOM.
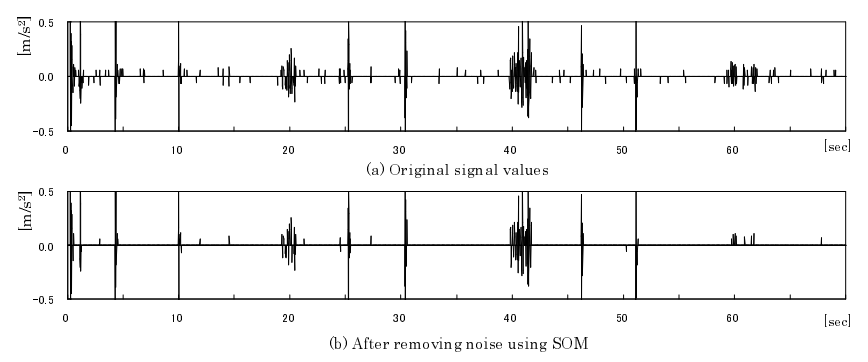

Figure 11. Noise removing result using SOM (Pillow sensor, $\mathrm{X}$ axis).

subject and each trial. Therefore, it is a challenging task to set thresholds for removal of noise or for recognition solely from original signals. In contrast, our machinelearning based method can execute the following process automatically.

\subsection{Noise removal results}

For improving recognition accuracy and to reduce processing costs together, we removed noise patterns using SOMs. Fig. 10 portrays a clustering result of training datasets using SOMs. The horizontal and vertical axes depict the position of a burst unit and the number of burst iterations, which correspond to the number of datasets in each unit. We sorted a unit's numbers according to the maximum of the bursting iterations from the left side. Most output voltages remained near the offset value shown in Fig. 9 because no output voltage when no load change is given without bending.

In Fig. 10, the sixth unit corresponds to this unit. For this case, 25,954 signals were mapped to the unit that corresponded to $74.2 \%$ for all data of 34,976 signals. Other signals of $15.8 \%$ were mapped to the remaining 19 units, similar to a long-tail shape. We confirmed that this tendency was obtained in other cases when we changed the number of units.

Fig. 11 portrays signal patterns of the $\mathrm{X}$ axis from the pillow sensor during the first $70 \mathrm{~s}$ period after removing signals mapped into the sixth unit. As the whole tendency, noise signals around $\pm 0.1\left[\mathrm{~m} / \mathrm{s}^{2}\right]$ were removed using our method. However, the signals around $60 \mathrm{~s}$ with higher amplitude were removed, while those with smaller amplitude were remained.

\subsection{Recognition results}

Fig. 12 depicts category maps of Subjects A, B, and $\mathrm{C}$ created as classifiers. The numbers on the category maps correspond to respective behaviors that were labeled though learning. Distributions of labels differ among subjects. Each category map created clusters of similar labels for similar behaviors.

We divided boundaries of units on the map with thick lines to depict segments of sleeping, sitting, and leaving, which are the global changes of behavior patterns. Similar behavior patterns created recognizable regions of behaviors. Units that are assigned to the seventh label as left from the bed are distributed in the cluster of units labeled sitting. We inserted dash marks in this figure to the label each subject. Using CPNs, our method created a classifier in each subject through learning. Moreover, our method enables visual confirmation of the relations among datasets.

Subsequently, we evaluated our method to recognize bed-leaving behaviors from matched labels that correspond to burst labels after presenting test datasets to the category maps. Table 2 portrays a result of recognition accuracy. We evaluated five combinations for CV. Herein, we calculated the recognition accuracy as a ratio of the number of ground truths and the number of labels in each behavior pattern that reached the maximum of responses during $20 \mathrm{~s}$.

The mean recognition accuracy for ten subjects is $75.5 \%$. The recognition accuracies of Subjects B and E are, respectively, $88.1 \%$ and $59.5 \%$ as the highest and the lowest. For respective behaviors, the recognition accuracy of leaving is $95.0 \%$. The recognition accuracy of lateral sitting remained at $53.3 \%$, although the recognition accuracies of longitudinal sitting and terminal sitting, which are the most important positions for bed-leaving detection, reached $83.3 \%$ and $98.3 \%$, respectively. For lateral sitting, the recognition accuracy of Subject B was $16.7 \%$. We regard lateral sitting as a status that can accommodate diversity among individuals. For sleeping, the recognition accuracy of face-up sleeping is higher than that of right or left sleeping. We regard the body weight as gathered to the two sensors of the left or right sides in sleeping, whereas the load from the upper body is distributed on $\mathrm{S} 1-\mathrm{S} 4$ at the face-up sleeping.

Herein, we compared the performance derived during a single use of pad sensors, a pillow sensor, and a bolt sensor. Table 3 portrays the recognition accuracies of respective sensors. The recognition accuracy of the pad sensors, which showed the highest performance, is $75.0 \%$. Comparison with our proposed multiple sensor system shows that the performance difference is $0.5 \%$. 


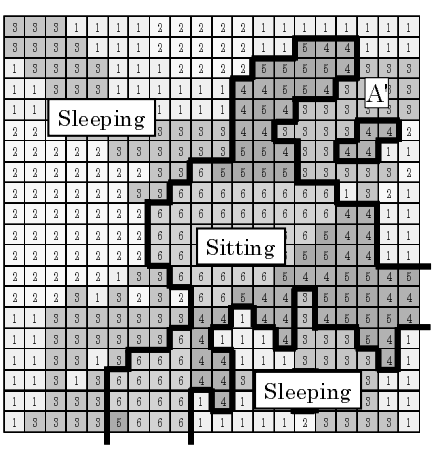

(a) Subject $\mathrm{A}$

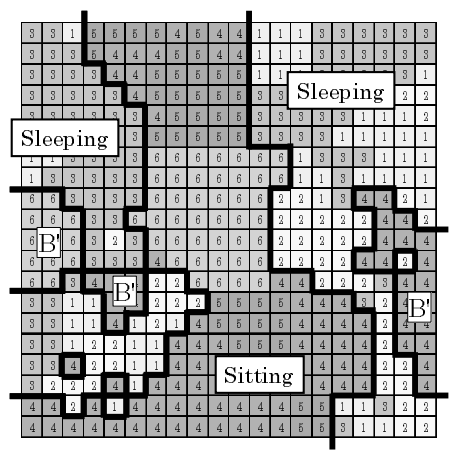

(b) Subject B

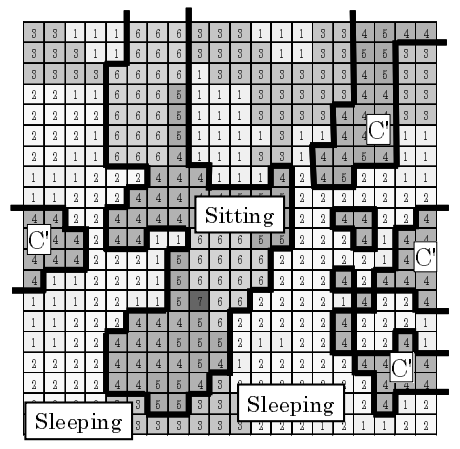

(c) Subject C

Figure 12. Categrory maps of Subjects A-C. The grids correspond to the units on the mapping layer of CPNs. Lavelts $1-7$ denote FU, RI, LE, LO, LA, TE, and LB.

Table 2. Recognition accuracies of respective subjects and positions for seven patterns [\%].

\begin{tabular}{c|c|c|c|c|c|c|c|c}
\hline Subject & FU & RI & LE & LO & LA & TE & LB & Average \\
\hline A & 100 & 83.3 & 83.3 & 100 & 33.3 & 100 & 100 & 85.7 \\
\hline B & 100 & 100 & 100 & 100 & 16.7 & 100 & 100 & 88.1 \\
\hline C & 0 & 66.7 & 100 & 100 & 83.3 & 100 & 100 & 78.6 \\
\hline D & 66.7 & 50.0 & 33.3 & 100 & 66.7 & 100 & 100 & 73.8 \\
\hline E & 50.0 & 83.3 & 16.7 & 50.0 & 33.3 & 83.3 & 100 & 59.5 \\
\hline F & 50.0 & 83.3 & 66.7 & 100 & 66.7 & 100 & 100 & 81.0 \\
\hline G & 66.7 & 66.7 & 50.0 & 66.7 & 50.0 & 100 & 83.3 & 69.0 \\
\hline H & 66.7 & 33.3 & 83.3 & 66.7 & 66.7 & 100 & 100 & 73.8 \\
\hline I & 66.7 & 50.0 & 66.7 & 66.7 & 66.7 & 100 & 100 & 73.8 \\
\hline J & 100 & 66.7 & 33.3 & 83.3 & 50.0 & 100 & 66.7 & 71.4 \\
\hline Ave. & 66.7 & 68.3 & 63.3 & 83.3 & 53.3 & 98.3 & 95.0 & 75.5 \\
\hline
\end{tabular}

Recognition accuracies used solely for a pillow sensor and a bolt sensor were, respectively, $58.3 \%$ and $33.3 \%$. During use setting priority to simplicity, we regard that it is possible to use each single sensor except for the bolt sensor. We regard our method for a multiple sensor system as useful for situations favoring performance over simplicity.

\subsection{Discussion}

Table 4 portrays the confusion matrix in each method and scene category for analyzing details of classification results. For this matrix, the number of correct images is shown on the diagonal cells that are marked as boiled numbers. Other cells refer the number of incorrect images and its category name shown in the column. The maximum numbers of incorrect categories are marked as underlined.

The recognition accuracy of lateral sitting is the second lowest. The number of correct recognition is 32 datasets. For this behavior, 20, 5, and 3 datasets are falsely recognized respectively as longitudinal sitting, terminal sitting, and left the bed. The behavior patterns of a subject from longitudinal sitting to lateral sitting have a large gap because subjects turn their body about $90 \mathrm{deg}$ on the bed. For both sitting positions, the load to be provided pad sensors is concentrated to the hip at the center of the bed. We consider that the false recognition in both behavior patterns results from this feature. For lateral sitting, the load of the legs is gath- ered to the terminal as an exit of the bed. We consider that false recognition results from unclear boundaries between lateral sitting and terminal sitting. However, we consider that fatal errors of bed-leaving prediction can be avoided because no dataset of lateral sitting was falsely recognized as sleeping or bed leaving.

As shown in the fourth line of Table 4, 12 and 9 datasets of left sleeping were falsely recognized as right sleeping and face-up sleeping, respectively. False recognition of left sleeping remains for sleeping except for one dataset. For face-up and right sleeping, four datasets are falsely recognized as longitudinal sitting. We consider that this results from the load to be gathered S3 only depending on a subject, although the load on right sleeping is gathered to S1 and S3 normally.

We examined recognition of three patterns: sleeping, sitting, and leaving. Table 5 presents the confusion matrix with respective recognition accuracies for this case. The recognition accuracy of sleeping improved to $93.9 \%$. For bed-leaving prediction, these are basic behavior patterns. Therefore, we consider that our system, although simple, provides high recognition accuracy of $97.8 \%$ for standing.

Comparison with the bed-leaving sensor system that used strain gauges in actuators proposed by Hatsukari et al. [17] revealed that the recognition accuracies of their method were $87.7 \%$ for longitudinal sitting and $98.1 \%$ for terminal sitting. The recognition accuracies of terminal sitting and longitudinal sitting of our method are, respectively, $0.2 \%$ higher and $4.4 \%$ lower than their 
Table 3. Recognition accuracies used for stand-alone sensor [\%].

\begin{tabular}{c|c|c|c|c|c|c|c|c}
\hline Sub. & FU & RI & LE & LO & LA & TE & LB & Average \\
\hline Pads & 35.0 & 81.7 & 75.0 & 90.0 & 55.0 & 96.7 & 91.7 & 75.0 \\
\hline Acc. & 68.0 & 34.0 & 46.0 & 52.0 & 46.0 & 74.0 & 88.0 & 58.3 \\
\hline Bolt & 33.3 & 0 & 11.1 & 0 & 22.2 & 100 & 66.7 & 33.3 \\
\hline
\end{tabular}

Table 4. Confusion matrix of respective positions for seven patterns. Underlines show the numbers of correct data.

\begin{tabular}{c|c|c|c|c|c|c|c}
\hline Position & FU & RI & LE & LO & LA & TE & LB \\
\hline FU & $\underline{\mathbf{4 0}}$ & 3 & 15 & 2 & 0 & 0 & 0 \\
\hline RI & 5 & $\underline{\mathbf{4 1}}$ & 6 & 2 & 0 & 6 & 0 \\
\hline LE & 9 & 12 & $\underline{\mathbf{3 8}}$ & 0 & 0 & 1 & 0 \\
\hline LO & 0 & 0 & 1 & $\underline{\mathbf{5 0}}$ & 7 & 2 & 0 \\
\hline LA & 0 & 0 & 0 & 20 & $\underline{\mathbf{3 2}}$ & 5 & 3 \\
\hline TE & 0 & 0 & 0 & 1 & 0 & $\underline{\mathbf{5 9}}$ & 0 \\
\hline LB & 0 & 0 & 0 & 3 & 0 & 0 & $\underline{\mathbf{5 7}}$ \\
\hline
\end{tabular}

Table 5. Confusion matrix with respective recognition accuracies for three patterns

\begin{tabular}{c|c|c|c|c}
\hline & Sleeping & Standing & Left & Acc. [\%] \\
\hline Sleeping & 169 & 11 & 0 & 93.9 \\
\hline Standing & 1 & 176 & 3 & 97.8 \\
\hline Left & 0 & 3 & 57 & 95.0 \\
\hline
\end{tabular}

method, although the experimental environment and the number of subjects differ in the results. Their method remains for three target patterns of longitudinal sitting, terminal sitting, and leaving. Moreover, their method requires setting the body weight of a subject in advance. We consider that our method is superior to their method as a functional aspect without setting it in advance.

\section{Conclusion}

This paper presented a multiple sensor system for predicting behavior patterns that occur when a person leaves a bed. This sensor system is designed for early prediction of bed-leaving behavior patterns, with high regard devoted to QOL. We developed a method using machine learning algorithms of two types to recognize bed-leaving behavior patterns from signals obtained using the sensors. We evaluated our system for ten subjects in an environment that represents a clinical site. The recognition accuracy for seven behavior patterns was $84.1 \%$. Moreover, the recognition accuracies for longitudinal sitting, terminal sitting, and left the bed were $83.3 \%, 98.3 \%$, and $95.0 \%$, respectively. However, falsely recognized patterns remained for respective categories of sleeping and sitting on the bed. Our system is applicable to an actual environment as a novel sensor system that does not restrict patients.

We will achieve steady detection to expand the application range of our method and thereby increase the number of subjects. Moreover, we would like to ap- ply our system to care facilities or single senior's homes for security and safety observation that simultaneously maintains QOL and privacy.

\section{Acknowledgements}

We would like to extend our appreciation to Assistant Professor M. Takahashi at the Medical School of Akita University, who provided us medical knowledge and evaluation methods of our sensor system from the viewpoint as a medical doctor. Moreover, we would like to thank ten people who cooperated as subjects to produce datasets for this study.

\section{REFERENCES}

[1] Inokuchi, K.: The Labor Shortage in Care Workplace and Employment Intention of the Young, Proc. Kanazawa Univ. Graduate School Human and SocioEnvironmental Studies, Vol.15, 69-84, 2008.

[2] R. Yamada, M. Takashima, Y. Sato, W. Ito, T. Ito, and Y. Asanuma, "Evaluation and Prevention of Inpatient Falls -A study using a classification system based on situational criteria-," Proc. Akita Univ. School of Health Sciences, vol.18, no.2, pp.144-150, 2010.

[3] Y. Mitadera and K. Akazawa, "Analysis of Incidents Occurring in Long-Term Care Insurance Facilities," Bulletin of Social Medicine, vol.30, no.2, pp.123-130, 2013.

[4] K. Imaizumi, Y. Iwakami, and K. Yamashita, "Availability of Monitoring System for Supporting Healthcare of Elderly People," Japanese Journal of Applied IT Healthcare, vol.5, no.1, pp.63-64, 2010.

[5] H. Matsuda, A. Yamaguchi, and T. Arakawa, "Monitoring System of Living Activities for Elderly People," National Technical Report, vol.82, pp.4-8, 2003.

[6] T. Tatsumi, K. Kanemoto, and N. Yagi, "How to Use Efficient Tentomushi - Considering the Length of Stride and Height for Protecting False Operation," Proc. Japanese Nursing Association, vol.38, pp.144$146,2007$.

[7] S. Kondo, C. Kamiya, H. Miyamoto, Y. Toriyama, E. Mimura, and F. Tsuchida, "Availability of Sensor Mats to Detect Leaving for Protection of Falling Accidents from Bed," Trans. Japanese Association of Rural Medicine, vol.55, no.3, pp.245, 2006.

[8] K. Haruyama, K. Tanaka, S. Kobayashi, K. Yasuoka, A. Uchibori, and M. Oka, "Development of Getting Up Detection and Report Device using Power Line Communication and Mat-Sensor," Trans. Institute of Electrical 
Engineers of Japan Part. D, vol.126, no.11, pp.15071513, 2006.

[9] H. Seki and Y. Hori, "Detection of Abnormal Action Using Image Sequence for Monitoring System of Aged People," Trans. Institute of Electrical Engineers of Japan Part. D, vol.122, no.2, pp.1-7, 2002.

[10] H. Madokoro, N. Shimoi, K. Sato, "Bed-Leaving Detection Using Piezoelectric Unrestrained Sensors and Its Measurement System Regarding QOL," Nursing and Health, vol.1, no.2, pp.36-45, 2013.

[11] M. Shimizu, K. Dugawara, F. Ozaki, Y. Hama, M. Nishimura, and H. Yoshino, "Development of Detection System of Getting out of Bed with Ultrasonic Array Sensor (Part 3)," Trans. Society of Life Support Technology, vol.21, no.3, pp.9-16, 2009.

[12] K. Hirasawa, N. Matsumura, N. Kanemaru, and K. Abe, "Falling Accident Prevention System Used for a Clinical Site and Care Facility," Technical Journal of Nippon Telegraph and Telephone, vol.20, no.1, pp.3235,2008 .

[13] T. Uezono, A. Kubo, M. Nakajo, A. Uekaraseta, and T. Uchida, "Application Study of Bed leaving Prediction System," Proc. Conference of Kagoshima Prefectural Industrial Technology Center, pp.34-35, 2010.

[14] H. Madokoro, N. Shimoi, and K. Sato, "Prediction of Bed-Leaving Behaviors Using Piezoelectric NonRestraining Sensors," Journal of Sensors and Sensor Systems, vol.2, no.1, pp.27-34, Apr. 2013.

[15] N. Shimoi and H. Madokoro, "Flight Stabilization of Micro Flying Robot for Rescue Scenarios," International Journal of Automation, Robotics and Autonomous Systems, vol.12, no.1, pp.11-15, Dec. 2012.

[16] N. Shimoi, C. H. Cuadra, H. Madokoro, and M. Saijo, "Simple Smart Piezoelectric Bolt Sensor for Structural Monitoring of Bridges," International Journal of Instrumentation Science, vol.1, no.5, pp.78-83, Dec. 2012.

[17] T. Hatsukari, T. Shiino, and S. Murai, "The Reduction of Tumbling and Falling Accidents Based on a Built-in Patient Alert System in the Hospital Bed," Journal of Science of Labour, vol.88, no.3, pp.94-102, 2012.

[18] Y. Kishimoto, A. Akahori, and K. Oguri, "Position presumption during sleeping by using a three-axis acceleration sensor," IEICE Technical Report of ME and Bio Cybernetics, vol.105no.456pp.45-48, 2005.

[19] K. Nomoto, R. Miyazaki, T. Hasegawa, and Y. Yonei, "A Trial of Evaluation for Quality of Sleep using a Three Dimension Acceleration Sensor," The Science and Engineering Review of Doshisha University, vol.51, no.1, pp.28-36, 2010.

[20] S. Okada, Y. Fujiwara, H. Matsuura, M. Yasuda, K. Sugai, M. Makikawa, and T. Iida, "Heart Beat Monitoring during Sleeping Using an Accelerometer Set inside the Coverlet," Transactions of the Japanese Society for Medical and Biological Engineering, vol.41, no.4, pp.493-497, 2003.
[21] Measurement Specialties Inc.: Datasheet ofDT Series Elements with Lead Attached, Rev.1, 2009.

[22] Freescale Semiconductor, MMA7361LC Data Sheet, Technical Data, Rev 1, 2011.

[23] Nakamura, K., Kakusho, K., Murakami, M. and Minoh, M.: Estimating Learners' Subjective Impressions of the Difficulty of Course Materials by Observing Their Faces in e-Learning, The IEICE Transactions on Information and Systems, J93-D, 5, pp.568-578, 2010.

[24] M. Motegi, N. Matsumura, T. Yamada, N. Muto, N. Kanamaru, K. Shimokura, K. Abe, Y. Morita, and K. Katsunishi, "Analyzing Rising Patterns of Patients to Prevent Bed-related Falls (Second Report)," Trans. Japan Society for Health Care Management, vol.12, no.1, pp.25-29, 2011.

[25] T. Kohonen, Self-Organizing Maps, Springer Series in Information Sciences", 1995.

[26] T. Yamakawa, H. Tokutaka, and K. Fujimura, Application Examples of Self-Organizing Maps, Kaibundo Pub., 2002.

[27] J. McQueen, "Some Methods for Classification and Analysis of Multivariate Observations", Proc. Fifth Berkeley Symposium on Mathematical Statistics and Probability, pp.281-297, 1967.

[28] J. Vesanto and E. Alhoniemi, "Clustering of the Self-Organizing Map", IEEE Trans. Neural Networks, vol.11, no.3, pp.586-600, 2000.

[29] M. Terashima, F. Shiratani, and K. Yamamoto, "Unsupervised Cluster Segmentation Method Using Data Density Histogram on Self-Organizing Feature Map," Trans. Institute of Electronics, Information and Communication Engineers, vol.J79-D-2, no.7, pp.1280-1290, 1996.

[30] H.B. Barlow, "Unsupervised learning," Neural Computation, no.1, pp.295-311, 1989.

[31] K. Doya, "What are the computations of the cerebellum, the basal ganglia, and the cerebral cortex," Neural Networks, vol.12, pp.961-974, 1999.

[32] H. Nielsen, "Counterpropagation networks", Proc. of IEEE First Int'l. Conf. on Neural Networks, 1987.

[33] M. Tsukada, Y. Utsumi, H. Madokoro, and K. Sato, "Unsupervised Feature Selection and Category Classification for a Vision-Based Mobile Robot", IEICE Trans. Inf. ES Sys., vol.E94-D, no.1, pp.127-136, 2011.

[34] M. Hosokawa, Y. Ito, and T. Hoshi, "Extraction of Urban Characteristics using Polarimetric SAR Data and Self-Organizing Map," Trans. Institute of Electronics, Information and Communication Engineers B, vol.J84B, no.6, pp.1043-1051, 2001.

[35] R. Kohavi, "A study of cross-validation and bootstrap for accuracy estimation and model selection", Proc. the Fourteenth International Joint Conference on Artificial Intelligence, vol.2, no.12, pp.1137-1143, 1995. 